\title{
STUDY OF THE ANTIDEPRESSANT ACTIVITY OF FOLIC ACID AND VITAMIN-D ON RESERPINE INDUCED DEPRESSION IN MICE
}

\author{
BORAH L, LAHKAR M, DASGUPTA S*
}

Department of Pharmacology, Gauhati Medical College, Guwahati, Assam, India. Email: shreyashi.dasgupta@gmail.com

Received: 24 October 2017, Revised and Accepted: 30 October 2017

\begin{abstract}
Objective: Low levels of folic acid and deficiency of Vitamin D have been found to be associated with poor mood and depression. This study was designed to investigate whether these vitamins show antidepressant activity in models of depression in mice.

Methods: Reserpine was used to induce depression in the study groups. Low and high doses of folic acid and Vitamin D as well as combinations of these vitamins in low and high doses were administered after induction of depression. The test animals were then tested on forced swim test, tail suspension test, and open field test models for evaluation of the antidepressant activity.
\end{abstract}

Results: After 2 weeks of drug treatment, all the treated groups showed a significant reduction in immobility time in both the test models (p<0.05). High dose folic acid showed consistently greater antidepressant property in all the test models throughout the study period. High dose Vitamin D $(\mathrm{p}<0.05)$ also showed good antidepressant activity after 2 weeks, the delayed antidepressant effect of which might be attributable to the molecular mechanism of action of Vitamin D.

Conclusion: Our study demonstrates that both folic acid and Vitamin D have antidepressant activity. The antidepressant activity of high dose folic acid $(50 \mathrm{mg} / \mathrm{kg})$ in reserpine-induced depression in mice at the end of 2 weeks was more pronounced in our study. Studies with longer duration of treatment are warranted to further evaluate their antidepressant effect.

Keywords: Folic acid, Vitamin D, Reserpine, Depression, Tail suspension test, Forced swim test.

(C) 2018 The Authors. Published by Innovare Academic Sciences Pvt Ltd. This is an open access article under the CC BY license (http://creativecommons. org/licenses/by/4. 0/) DOI: http://dx.doi.org/10.22159/ajpcr.2018.v11i2.20408

\section{INTRODUCTION}

Depression is a condition characterized by depressed mood, loss of interest or loss of pleasure in nearly all activities, most of every day, for a period lasting at least 2 weeks [1]. It is commonly associated with easy fatigability, irritability, feelings of worthlessness, guilt, changes in sleep pattern, and suicidal ideation. The Global Burden of Disease study cites depression as one of the leading causes of disability affecting 350 million people worldwide [2]. The incidence of major depressive disorder is found to be more in women (10-25\%) than men (5-12\%). Recurrence is also fairly common with an incidence of $50-60 \%$ in those who have experienced a single depressive episode. Depression is also found to be accompanied by anxiety [3].

Several genetic and epigenetic factors are known to be potential causes of depression, some of which have already been identified. Current research work has mainly focused on two hypotheses regarding the pathophysiology of depression. First, the monoamine deficiency hypothesis links depression to the availability and function of monoamine neurotransmitters in the brain, of which serotonin and norepinephrine have been studied in the greatest detail. A second, related hypothesis for the development of depression, involves the impact of stress on psychological functioning. In response to stress, the hypothalamus releases corticotropin-releasing hormone, which stimulates the pituitary to release corticotropins, which in turn stimulates the adrenals to release cortisol. Impairment in the functioning of the hypothalamic-pituitary-adrenal axis may be directly involved in depression as well as anxiety, but as with monoamines, the relationship is complex [4].

Folic acid has been shown to have a role in serotonin metabolism by acting as a methyl donor for the conversion of methionine to S-adenosyl methionine. It also has an important role in the synthesis of tetrahydrobiopterin, an essential cofactor for the hydroxylation of phenylalanine and tryptophan, rate-limiting steps in the synthesis of dopamine (DA), noradrenaline (NA), and serotonin (5-HT) [5,6].

Vitamin D receptors (VDR) have been recently identified in several tissues of the body, including both neuronal and glial cells in the central nervous system [7]. VDRs have been identified in multiple areas of the human brain, including the prefrontal cortex, hippocampus, cingulate gyrus, thalamus, hypothalamus, and substantia nigra, [8] many of which have been implicated in the pathophysiology of depression [9]. Other studies have suggested that low levels of Vitamin D are associated with poor mood. A number of trials have suggested a role for Vitamin D in the supplementary treatment of depression. Dose may be a critical issue, as sun exposure and dietary intake may be low and high doses may be required [10].

Monoamines have been implicated in the pathogenesis of depression, and folic acid is closely involved in their biosynthesis. Deficiency of Vitamin D has also been associated with poor mood. Both these vitamins are widely used for managing various clinical conditions, and their safety is well established. We, therefore, conducted this study with the hypothesis that folic acid and Vitamin D administration produces antidepressant-like effects. Considering the paucity of preclinical studies dealing with the antidepressant-like effects of folic acid and Vitamin D, this study sought to investigate the effect of these vitamins in models of depression in mice.

\section{AIMS AND OBJECTIVES}

1. To evaluate the antidepressant effect of low dose and high dose of folic acid.

2. To evaluate the antidepressant effect of low dose and high dose of Vitamin D. 
3. To evaluate the antidepressant effect of combinations of folic acid and Vitamin D, in low and high doses, respectively.

4. To compare the antidepressant effects of folic acid, Vitamin D and combination of folic acid and Vitamin D with a standard drug of depression, fluoxetine.

\section{MATERIALS AND METHODS}

This study was conducted in the Department of Pharmacology, Gauhati Medical College, Guwahati, for a period of 3 months from July 2016 to September 2016 after taking due approval from the Institutional Animal Ethics Committee (MC/5/2015/82).

Male Swiss Albino mice (26-32 g), obtained from the College of Veterinary Sciences, Khanapara, Guwahati, were used for this study. The animals were acclimatized to laboratory conditions for 7 days before commencement of the experiment. The animals were housed at room temperature, subjected to $12 \mathrm{~h}$ light and $12 \mathrm{~h}$ dark cycles and were fed with standard diet and water ad libitum. Mice belonging to the extremes of age, female and diseased mice were excluded from the study. All the experiments were performed according to the guidelines of the Committee for the Purpose of Control and Supervision of Experiments on Animals, Government of India.

A total of 54 animals meeting the inclusion criteria were randomly selected and were divided into the following groups, each containing a total of 6 animals.

Normal saline (normal control), reserpine only (disease control), reserpine + fluoxetine $20 \mathrm{mg} / \mathrm{kg}$ (standard control), reserpine + folic acid low dose (FALD), reserpine + folic acid high dose (FAHD), reserpine + Vitamin D low dose (VDLD), reserpine + Vitamin D high dose (VDHD), reserpine + FALD + VDLD, and reserpine + FAHD + VDHD.
Reserpine $1 \mathrm{mg} / \mathrm{kg}$ (Sigma-Aldrich, St. Louis, MO, USA), freshly prepared in distilled water was administered intraperitoneally from Day 1 to 3 to induce depression. Folic acid (low dose: $10 \mathrm{mg} / \mathrm{kg}$; high dose: $50 \mathrm{mg} / \mathrm{kg}$ ) [11] was administered per orally from day 7 to 20 . Vitamin $\mathrm{D}_{3}$ (low dose: $0.2 \mu \mathrm{g} / \mathrm{kg}$; high dose: $5 \mu \mathrm{g} / \mathrm{kg}$ ) [12] dissolved in Tween 80 (Merck, Germany) was administered by intraperitoneal injection from day 7 to 20 .

The animals from all the groups were tested on three models, namely, forced swim test (FST), tail suspension test (TST), and open field test (OFT). TST and FST were conducted on the following days: Day 0 before commencing the experiments, day 4 - at the end of induction phase for depression, day 14 - midway through the treatment phase, and day 22 - at the end of the treatment phase. OFT was conducted on day 22 after the end of the treatment phase.

Results were expressed as mean \pm standard error of mean (SEM). Data were analyzed by using one-way analysis of variance, and the post-hoc analysis was performed using Tukey's multiple comparison tests. $\mathrm{p}<0.05$ was considered to be statistically significant. All statistical analysis was performed using GraphPad Prism version 5.01 for Windows.

\section{RESULTS}

At the start of the experiment (day 0), there was no significant difference in the period of immobility among the study groups in the TST and FST (Fig. 1a and b).

Following reserpine administration for 3 consecutive days, on day 4, the period of immobility increased significantly in all the reserpine-treated groups $(\mathrm{p}<0.05)$ in both the test models (Fig. $2 a$ and b).

Midway through treatment with low doses, high doses, and combination of folic acid and Vitamin D, on day 14, the duration of immobility was found to be significantly decreased in all the groups receiving treatment

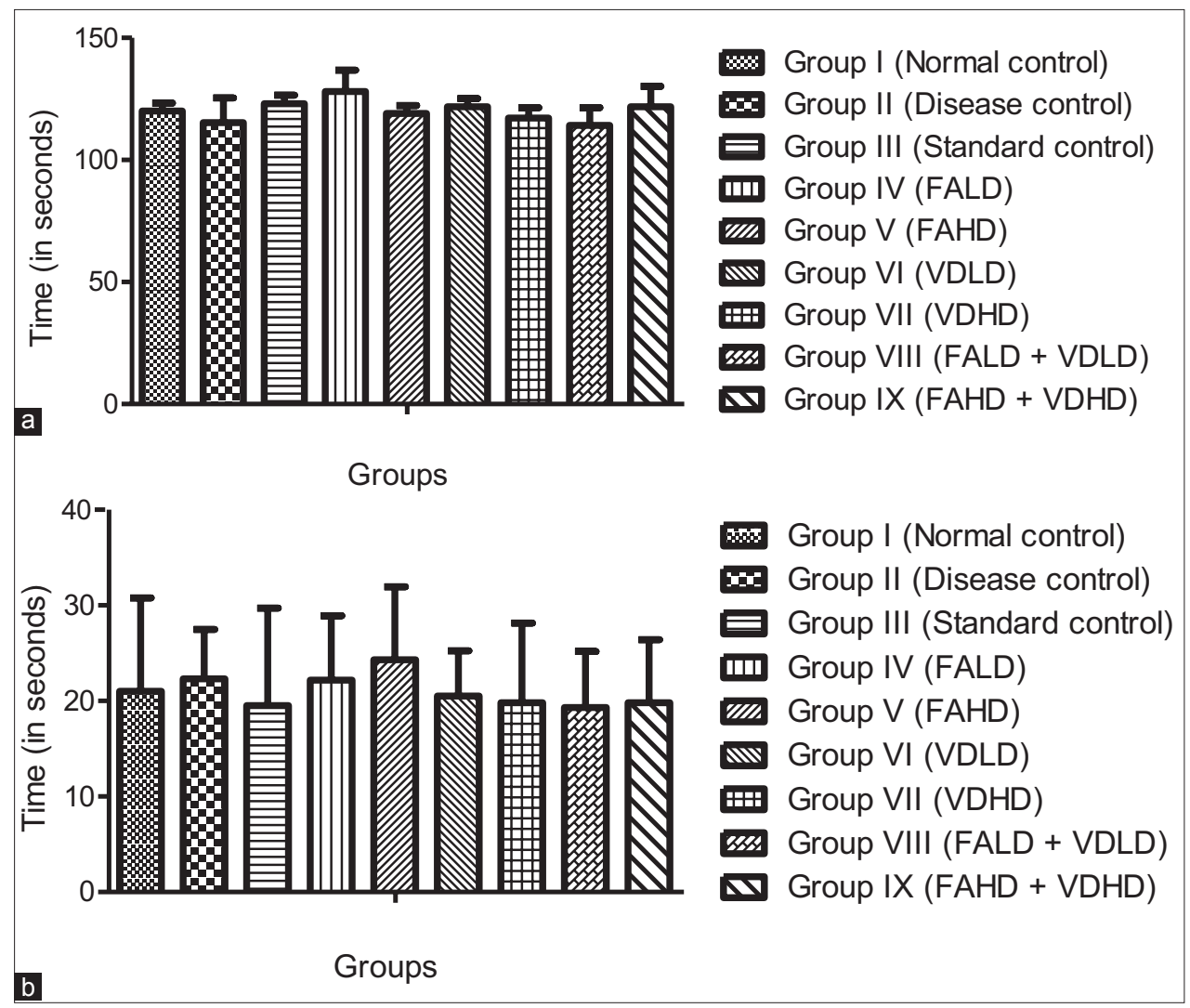

Fig. 1a: Tail suspension test results on day 0 . Values are expressed as a mean \pm standard error of mean $(n=6)$, (b) forced swim test results on day 0 . Values are expressed as mean \pm standard error of mean $(n=6)$ 
with folic acid or Vitamin D or both in the TST. In addition, the immobility period in only the FALD group was found to be significantly higher as compared to the standard control group, though it was lower than the normal control (Fig. 3a).

In the FST, on day 14, all the groups were significantly higher than the normal control. All the treatment groups except the VDLD group showed significantly lower immobility periods than the standard control, the decrease being significantly lower in the FAHD group as compared to the standard drug, fluoxetine (Fig. 3b).

At the end of the treatment phase, on day 22, the period of immobility was decreased in all the treatment groups in TST. It was least in the group receiving high dose combination of folic acid and Vitamin D, which was significantly lower than both the normal control group and the standard control group (Fig. 4a).

On day 22, in the FST, significant reduction in immobility period was observed in all the treated groups except the groups receiving low dose folic acid and low dose Vitamin D. There was no statistically significant difference between the normal control group and the groups treated with high dose folic acid and combination of high doses of folic acid and Vitamin D. The immobility period in both FAHD group and high dose combination group was lower than the standard control group but only the FAHD group was significantly lower than the standard (Fig. 4b).

In the OFT conducted on day 22, total time spent in central and peripheral squares was significantly more in all the treated groups as compared to the group receiving standard drug (Fig. 5).

\section{DISCUSSION}

The present study was conducted to evaluate whether folic acid and Vitamin D have antidepressant effects and to explore the effects of various doses and combinations of these two drugs in reserpineinduced depression

Repeated administration of reserpine has been found to be a progressive model of depression and was used to induce depression in our study [13]. Fluoxetine, a selective serotonin reuptake inhibitor, was taken as the standard drug because it is known to reduce the immobility period of mice in TST and FST [14]. TST and FST are behavioral models of depression and can predict the antidepressant property of several drugs [15]. Both these models were used in our study to evaluate the antidepressant activity of folic acid and Vitamin D.

Depression was found to be induced in all the groups treated with reserpine $1 \mathrm{mg} / \mathrm{kg}$. After 1 week of drug treatment, all the treated groups showed a significant reduction in immobility period in TST and FST, except VDLD group in FST. In the TST, all the treated groups showed reduction in immobility which was comparable to the standard drugtreated group. Only the low dose folic acid group was an exception. In the FST on the other hand, high dose folic acid was found to produce a statistically significant decrease in immobility period, which was better than the response obtained by the standard drug. Therefore, after 1 week of treatment, amelioration of depression was consistently observed with a daily dose of folic acid $50 \mathrm{mg} / \mathrm{kg}$ in both the test models, and it showed better results than fluoxetine $20 \mathrm{mg} / \mathrm{kg}$ in reducing depression. Folic acid $10 \mathrm{mg} / \mathrm{kg}$ and both the low dose and high dose combinations of folic acid and Vitamin D showed anti-depressant effect in one test model, TST.

After 2 weeks of drug treatment, all the treated groups showed a significant reduction in immobility time which was comparable to that seen in the normal control group in TST. Maximum reduction in immobility time was observed in the group receiving a combination of high dose Vitamin D and folic acid. In FST also, all treated groups showed antidepressant activity except low dose folic acid. High dose

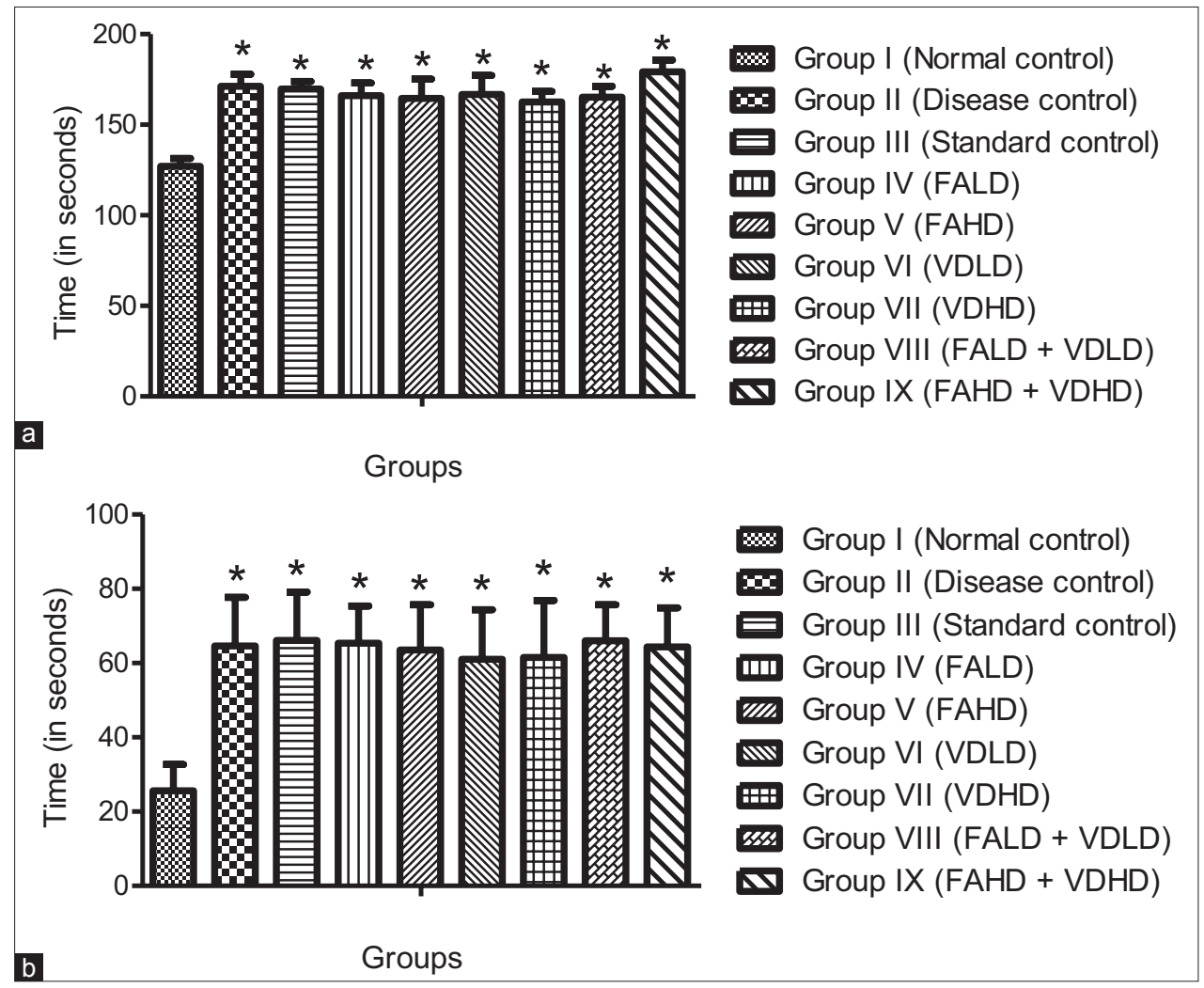

Fig. 2: (a) Tail suspension test results on day 4. Values are expressed as a mean \pm standard error of mean ( $=6$ ). * $p<0.05$ as compared to normal control group, (b) forced swim test results on day 4 . Values are expressed as a mean \pm standard error of mean ( $\mathrm{n}=6$ ). ${ }^{*} \mathrm{p}<0.05$ as compared to normal control group 


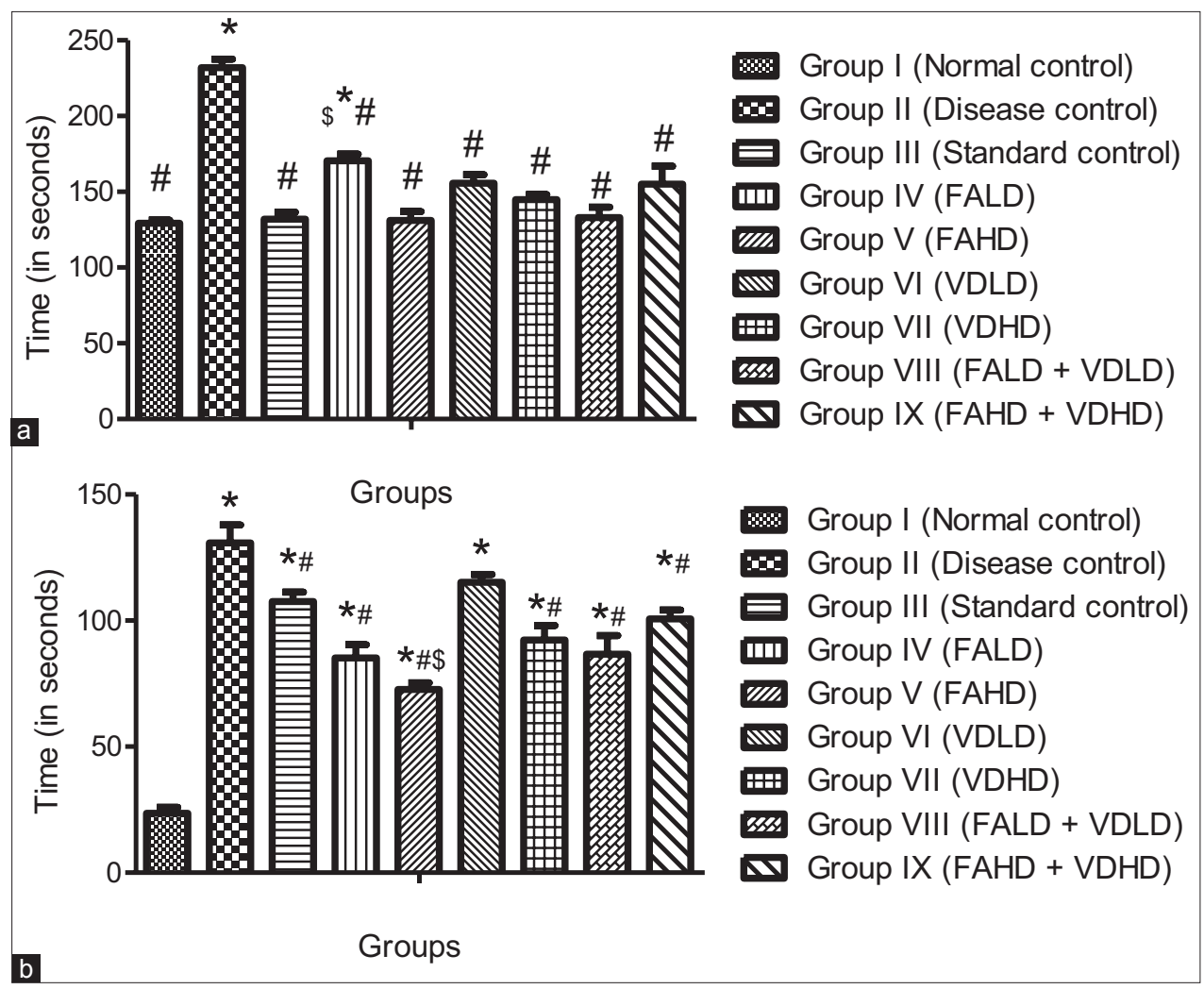

Fig. 3: (a) Tail suspension test results on day 14. Values are expressed as a mean \pm standard error of mean ( $n=6)$; ${ }^{*} \mathbf{p}<0.05$ as compared to normal control group, $\# \mathbf{p}<0.05$ as compared to disease control group, $\$ \mathrm{p}<0.05$ as compared to standard control group, (b) forced swim test results on day 14 . Values are expressed as mean \pm standard error of mean $(n=6) ;{ }^{*}<<0.05$ as compared to normal control group, $\# p<0.05$ as compared to disease control group, $\$ p<0.05$ as compared to standard control group

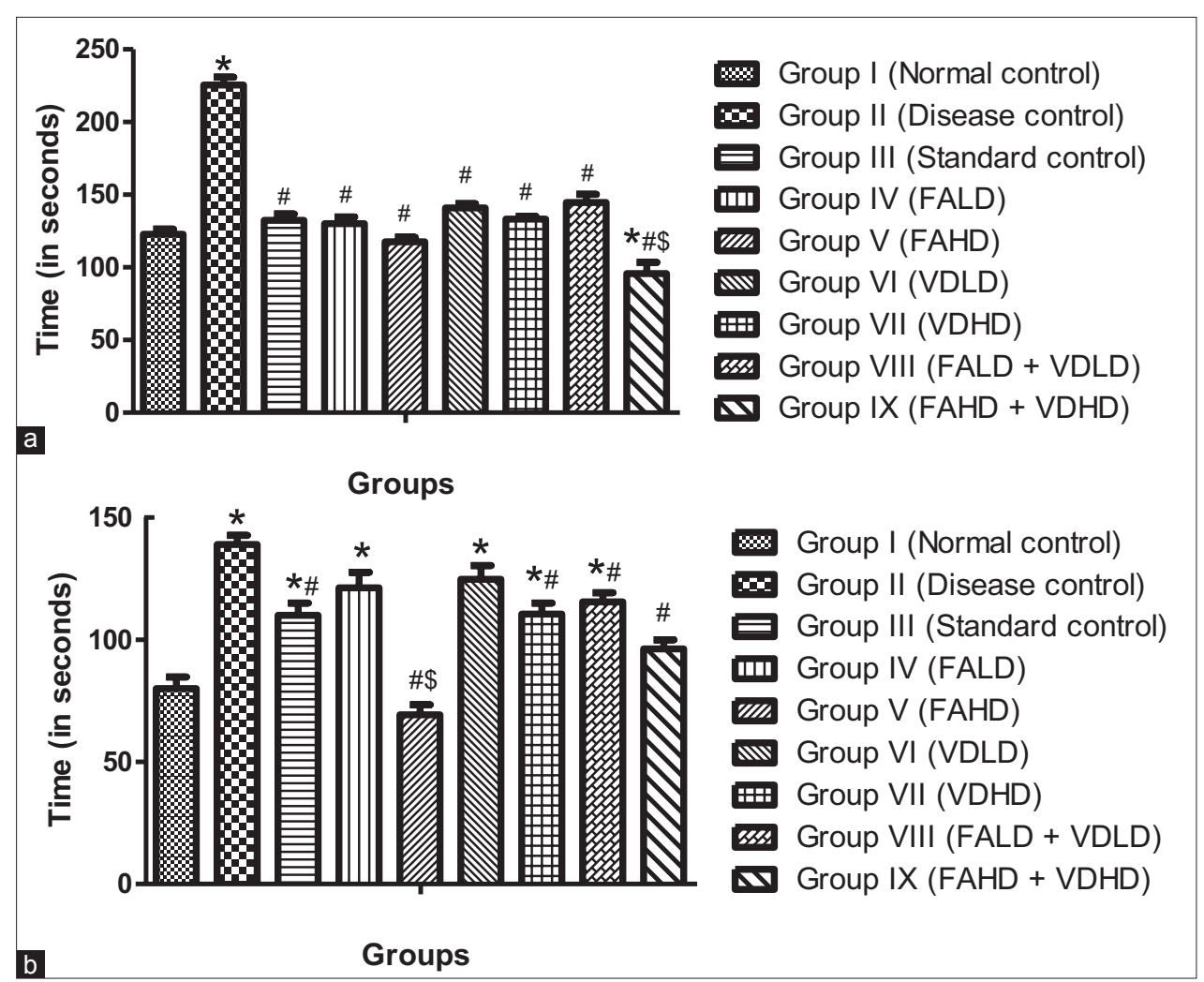

Fig. 4: (a) Tail suspension test results on day 22. Values are expressed as a mean \pm standard error of mean ( $\mathrm{n}=6)$; ${ }^{*} \mathrm{p}<0.05$ as compared to normal control group, $\# \mathbf{p}<0.05$ as compared to disease control group, $\$ \mathrm{p}<0.05$ as compared to standard control group, (b) forced swim test results on day 22 . Values are expressed as mean \pm standard error of mean $(n=6) ;{ }^{*} p<0.05$ as compared to normal control group, $\# p<0.05$ as compared to disease control group, $\$ p<0.05$ as compared to standard control group 


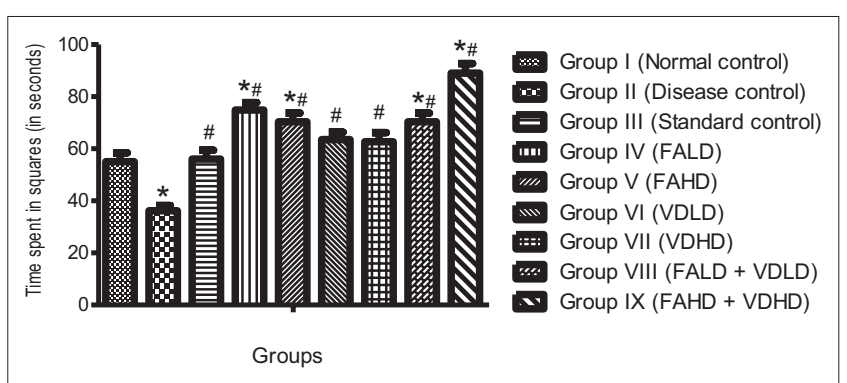

Fig. 5: Open field test results on day 22. Values are expressed as mean \pm standard error of mean $(n=6) ;{ }^{*} p<0.05$ as compared to normal control group, $\# \mathbf{p}<0.05$ as compared to disease control group

combination group and FAHD group had no significant difference in immobility time with the normal control group. High dose folic acid showed significantly greater antidepressant activity than the standard fluoxetine-treated group. Results of OFT also imply improved general locomotor activity and lesser anxiety in the groups receiving drug treatment.

Our study corroborates the findings of other authors on the antidepressant activity of folic acid and Vitamin D. Brocardo et al. demonstrated an antidepressant-like effect of folic acid in FST and TST, and they ascribed this effect to an interaction with the serotonergic and noradrenergic systems [11]. Budni et al. in their study found that folic acid (50 mg/kg, p.o.) was able to prevent the stress-induced increase in immobility time in the FST, but did not prevent memory impairment [16]. Fedotova et al. tested ovariectomized female rats on FST and OFT and found that cholecalciferol in high dose has a marked antidepressant-like effect in the adult female rats with low levels of estrogen [17].

\section{CONCLUSION}

The present study demonstrates that both folic acid and Vitamin D have antidepressant activity. The antidepressant activity of high dose folic acid $(50 \mathrm{mg} / \mathrm{kg}$ ) in reserpine-induced depression in mice was more pronounced in our study. This activity manifested within 1 week of initiating drug treatment and was sustained till the treatment ended. The rest of the treatment groups also showed improvement in depression, and after 1 week, combination therapy of high dose folic acid and Vitamin D showed results comparable to normal control. This might indicate that the antidepressant activity of Vitamin D monotherapy is slower to take effect, which might be because Vitamin D manifests most of its effects by transcription of necessary genes. Both folic acid and Vitamin D also demonstrated better effects on the general locomotor behavior and anxiety in the tested animals.

Our study had certain limitations. The treatment with folic acid and vitamin D was administered for a total period of 2 weeks only. Therefore, studies with longer duration of treatment are warranted to further evaluate their antidepressant effect.

\section{AUTHORS' CONTRIBUTIONS}

Study conception and design: Lahkar M, Borah L. Acquisition of data: Dasgupta S, Borah L, Lahkar M. Analysis and interpretation of data: Dasgupta S, Borah L, Lahkar M. Drafting of manuscript: Dasgupta S, Borah L. Critical revision: Dasgupta S, Borah L, Lahkar M.

\section{CONFLICT OF INTEREST}

The authors have no conflicts of interest to disclose.

\section{REFERENCES}

1. American Psychiatric Association. Diagnostic and Statistical Manual of Mental Disorders. $5^{\text {th }}$ ed. Washington, DC: American Psychiatric Association; 2013.

2. Zam WI. Vitamin D deficiency and depressive disorders: Review study of probable relationship. Int J Pharm Pharm Sci 2016;8:16-22.

3. Cerdá M, Sagdeo A, Galea S. Comorbid forms of psychopathology: Key patterns and future research directions. Epidemiol Rev 2008;30:155-77.

4. Belmaker RH, Agam G. Major depressive disorder. N Engl J Med 2008 3;358:55-68.

5. Mattson MP, Shea TB. Folate and homocysteine metabolism in neural plasticity and neurodegenerative disorders. Trends Neurosci 2003;26:137-46

6. Taylor MJ, Carney SM, Goodwin GM, Geddes JR. Folate for depressive disorders: Systematic review and meta-analysis of randomized controlled trials. J Psychopharmacol 2004;18:251-6.

7. Garcion E, Wion-Barbot N, Montero-Menei CN, Berger F, Wion D. New clues about Vitamin D functions in the nervous system. Trends Endocrinol Metab 2002;13:100-5.

8. Eyles DW, Smith S, Kinobe R, Hewison M, McGrath JJ. Distribution of the Vitamin D receptor and 1 alpha-hydroxylase in human brain. J Chem Neuroanat 2005;29:21-30.

9. Drevets WC, Price JL, Furey ML. Brain structural and functional abnormalities in mood disorders: Implications for neurocircuitry models of depression. Brain Struct Funct 2008;213:93-118

10. Berk M, Sanders KM, Pasco JA, Jacka FN, Williams LJ, Hayles AL, et al. Vitamin D deficiency may play a role in depression. Med Hypotheses 2007;69:1316-9.

11. Brocardo PS, Budni J, Kaster MP, Santos AR, Rodrigues AL. Folic acid administration produces an antidepressant-like effect in mice: Evidence for the involvement of the serotonergic and noradrenergic systems. Neuropharmacology 2008;54:464-73.

12. Tan ZX, Chen YH, Xu S, Qin HY, Zhang C, Zhao H, et al. Calcitriol inhibits bleomycin-induced early pulmonary inflammatory response and epithelial-mesenchymal transition in mice. Toxicol Lett 2016;240:161-71.

13. Ikram $H$, Haleem DJ. Repeated treatment with reserpine as a progressive animal model of depression. Pak J Pharm Sci 2017;30:897-902.

14. Walia V. Influence of stress and fluoxetine on immobility period of mice in tail suspension test and forced swim test. Asian J Pharm Clin Res 2016;9:302-5

15. Patel S, Rajshree N, Shah P. Evaluation of antidepressant activity of herbomineral formulation. Int J Pharm Pharm Sci 2016;8:145-7.

16. Budni J, Zomkowski AD, Engel D, Santos DB, dos Santos AA, Moretti $\mathrm{M}$, et al. Folic acid prevents depressive-like behavior and hippocampal antioxidant imbalance induced by restraint stress in mice. Exp Neurol 2013;240:112-21.

17. Fedotova J, Dudnichenko T, Kruzliak P, Puchavskaya Z. Different effects of Vitamin D hormone treatment on depression-like behavior in the adult ovariectomized female rats. Biomed Pharmacother 2016;84:1865-72. 